\title{
Olfactory Hallucination
}

National Cancer Institute

\section{Source}

National Cancer Institute. Olfactory Hallucination. NCI Thesaurus. Code C118175.

Perception of a smell in the absence of a corresponding stimulus. 